\title{
Multiple Tune Jumps to Overcome Horizontal Depolarizing Resonances
}

\author{
H. Huang, L. A. Ahrens, M. Bai, K. A. Brown, Y. Dutheil, C. Gardner, J. W. Glenn, \\ F. Lin, W. W. MacKay, F. Meot, A. Poblaguev, V. Ranjbar, T. Roser, V. Schoefer, \\ S. Tepikian, N. Tsoupas, K. Yip, A. Zelenski and K. Zeno \\ Brookhaven National Laboratory, Upton, New York 11973, USA \\ huanghai@bnl.gov
}

Published 29 February 2016

\begin{abstract}
Imperfection and vertical intrinsic depolarizing resonances have been overcome by the two partial Siberian snakes in the Alternative Gradient Synchrotron(AGS). The relatively weak but numerous horizontal resonances are the main source of polarization loss in the AGS. A pair of horizontal tune jump quads have been used to overcome these weak resonances. The locations of the two quads have to be chosen such that the disturbance to the beam optics is minimum. The emittance growth has to be mitigated for this method to work. In addition, this technique needs very accurate jump timing. Using two partial Siberian snakes, with vertical tune inside the spin tune gap and $80 \%$ polarization at AGS injection, polarized proton beam had reached $1.5 \times 10^{11}$ proton per bunch with $65 \%$ polarization. With the tune jump timing optimized and emittance preserved, more than $70 \%$ polarization with $2 \times 10^{11}$ protons per bunch has been achieved.
\end{abstract}

Keywords: Polarized proton; depolarizing resonance; spin.

PACS numbers: 41.75.Ak, 29.27.Bd, 29.27.Hj

\section{Introduction}

Polarized proton beams encounter many depolarizing resonances during acceleration in synchrotrons. In the past twenty years, Siberian snake ${ }^{1}{ }^{2}$ magnets have been invoked to overcome the depolarizing resonances. Since a full snake generates intolerable orbit distortion in the medium energy synchrotron, a partial snake ${ }^{3}{ }^{4}$ is the choice for medium energy range $(5-25 \mathrm{GeV})$. A dual partial snake scheme ${ }^{5}$ has been used in the AGS to overcome both imperfection and vertical intrinsic resonances.

For normal synchrotrons, the intrinsic resonance is only associated with the vertical betatron tune $\nu_{y}$ for vertical polarization, as the vertical spin can only be

*Current address: Jefferson Lab, Newport News, Virgina 23606, United States.

This is an Open Access article published by World Scientific Publishing Company. It is distributed under the terms of the Creative Commons Attribution 3.0 (CC-BY) License. Further distribution of this work is permitted, provided the original work is properly cited. 


\section{H. Huang et al.}

affected by the horizontal magnetic field. However, in the presence of a partial snake, the stable spin direction is not purely vertical. Therefore the perturbing fields that rotate the spin away from the stable spin direction have vertical as well as horizontal components. Particles undergoing horizontal betatron oscillations encounter vertical field deviations at the horizontal oscillation frequency. As a result, resonances with the spin tune are driven by the horizontal betatron oscillations, and will occur whenever the spin tune satisfies $G \gamma=k \pm \nu_{x}$, where $G$ is the anomalous magnetic moment factor of the beam particles, $\gamma$ is Lorentz factor, $k$ is an integer and $\nu_{x}$ is horizontal betatron tune. ${ }^{6}$

\section{Horizontal Tune Jump Scheme}

These horizontal intrinsic resonances can be divided into two categories: one with $M+\nu_{x}$; one with $K-\nu_{x}$. To increase the crossing speed, the horizontal tune is jumped up (so that $K-\nu_{x}$ going down) when crossing the $K-\nu_{x}$ resonance. It is then jumped down next when crossing $M+\nu_{x}$. Note that the associated vertical tune jump is in the opposite direction of horizontal tune jump. Since $\beta_{y}$ is about half of $\beta_{x}$ at the jump quad location, the vertical tune shift will be half the amplitude of horizontal one. When $G \gamma$ is near integer where imperfection and vertical intrinsic resonances are located, the vertical tune can be back to its nominal value, so that the crossings of these resonances are not affected, as illustrated in Fig. 1. With partial snakes inserted, the spin tune $\nu_{s p}$ is almost linearly proportional to $G \gamma$ except when $G \gamma$ is near an integer, where a spin tune gap is generated. There are two horizontal intrinsic and two vertical intrinsic resonances for each $G \gamma$ integer interval. The two vertical resonances are avoided by maintaining the vertical tune inside the spin tune gap. The two horizontal intrinsic resonances are overcome by tune jumping.

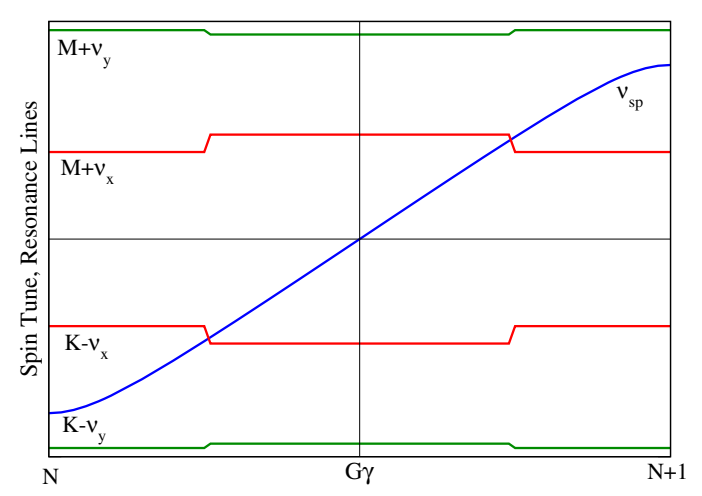

Fig. 1. The schematics of tune jump scheme. Each trace is labled. Over one unit of $G \gamma$, two horizontal intrinsic resonances are crossed. $\mathrm{M}, \mathrm{K}$ and $\mathrm{N}$ are integers. 
During normal acceleration in the AGS, the rate of change of $G \gamma$ is about $117 / \mathrm{s}$. The overall polarization loss due to horizontal intrinsic resonances for typical AGS emittance is about 15-20\% over the whole ramp. An increase of the crossing rate by 4 folds will reduce this loss to $4-5 \%$. The gain from further increase is small. To quadruple the resonance crossing speed, a change of $\nu_{x}$ at rate above $0.35 / \mathrm{ms}$ meets the requirement. The AGS horizontal tune jump system can achieve this with a tune jump of 0.04 in $100 \mu \mathrm{s}$.

Compared to vertical tune jump done in the past for polarization preservation, there are a few differences. First, this tune jump system has to jump many times (up to 82 times for the full AGS ramp), and the effect on polarization from each one is too small to be measurable. This means that the timing has to be dead reckoned. In the best scenario, we can only measure polarization as a function of the overall timing for all resonances. Second, the beam is used for collider injection instead of slow extracted beam. The emittance preservation is important for both polarization preservation and luminosity in the collider.

Simulations have been done for the possible emittance growth with tune jump. It was found out that two quadrupoles are better than just one quadrupole. The two quadrupoles are separated by phase advance of nearly $270^{\circ}$. Equal tune jump amplitude from the two quadrupoles is a condition for minimum emittance growth. With $20 \%$ beta function beat included, the vertical emittance growth is about two times larger than without the beta function beat. But it is no more than $8 \%$ for the total of 82 tune jumps.

During the beam test, the first observation was that the required currents for the two jump quads differed by a ratio of 3, which implies a big difference in beta functions at the two locations. The explanation is that a strong $18 \theta$ (two times the tune) vertical beta function beat was created because the tune is close to 9 and the synchrotron superperiod is 12 and not perfect. The effect is well modeled by adding an appropriately (but not uniquely) placed quadrupole into the lattice. The jump quads spacing ( 1 superperiod, $1 / 12$ of the ring) is $3 / 2$ of an $18 \theta$ wave. If one quad gets a bigger beta, the other gets a smaller beta.

All these effects can be dramatically reduced by adding some $6 \theta$ horizontal dipole component to the lattice which adds $18 \theta$ quad moment to the lattice by "feed down" in the sextupoles. With this addition, the jump quads gave equal tune shifts for equal currents and orbit response measurements confirm that the beta function beat is substantially reduced. With the orbit feed forward, removing the 6 th horizontal harmonic and proper chromaticity setup, the emittance is preserved for the horizontal tune jump.

\section{Horizontal Tune Jump Results}

The polarization coming into AGS is about $80-82 \%$. The details of the setup is in Ref.. ${ }^{7}$ The measured bunch length and synchrotron frequency give a constant longitudinal emittance of $0.76 \mathrm{eVs}$. This corresponds to dp/p FWHM as $2 \times 10^{-3}$ 


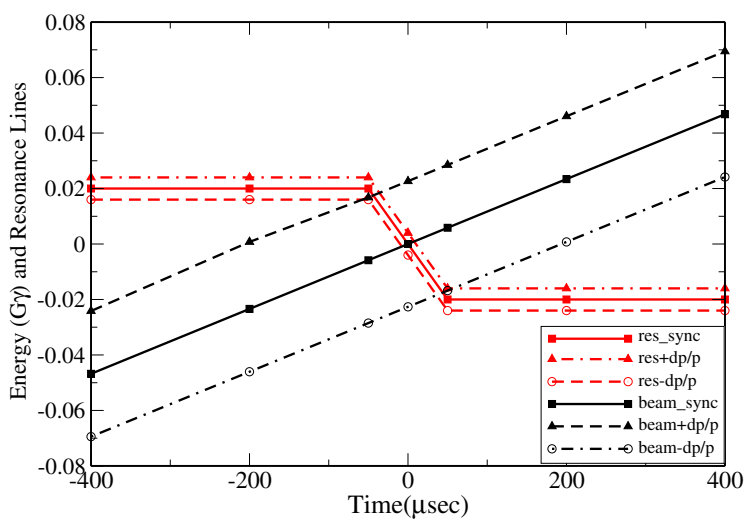

Fig. 2. Sketch of a spin resonance crossing on the increasing $\nu_{x}$ side of the pulse $(G \gamma=54-$ $\left.\nu_{x}=45.3\right)$. The horizontal axis is relative time to the resonance crossing. The vertical axis is energy relative to the synchronous particle at jump time in unit of $G \gamma$. The solid line applies to the synchronous particle, and the two types of broken lines apply to the boundary particles at the \pm FWHM. The resonance lines (red) are given by Eq.(1) for positive and negative momentum spreads. The momentum FWHM width is assumed as $10^{-3}$, which is close to the real dp/p at late part of the ramp. $\xi_{x}$ of -8 is used for the plot. The ramp rate $d G \gamma / d t$ is $0.117 / \mathrm{ms}$. The plot shows that particles within FWHM ( $76 \%$ beam) benefit from the tune jump in this case.

at injection and as $1 \times 10^{-3}$ at flattop. Note that $\mathrm{d} G \gamma$ is relatively flat around 0.04 above transition (300ms from T0), which is important to the tune jump system. In the following discussion, we consider beam particles inside the FWHM of the bunch, as about $76 \%$ of beam falls within this width for a Gaussian distribution.

In Fig. 1, each resonance was drawn as one line. With non-zero chromaticity and momentum spread, the resonance conditions should be rewritten as

$$
\begin{aligned}
& G \gamma=N-\left(\nu_{x} \pm \xi_{x} d p / p\right), \\
& G \gamma=N+\left(\nu_{x} \pm \xi_{x} d p / p\right),
\end{aligned}
$$

where $\xi_{x}$ is the horizontal chromaticity, $\pm d p / p$ gives the up and down boundaries of the momentum distribution. Ideally, zero chromaticity is needed to eliminate the tune (and resonance condition) dependence on momentum. This is more important for the vertical plane, as vertical intrinsic resonances are stronger. Even though the vertical tune is put into the spin tune gap, a small tune spread is still required as the higher order partial snake resonances limit the available tune space.

In the AGS, the vertical chromaticity is held near zero to reduce vertical tune spread. Putting both chromaticities near zero results in unstable beam. The horizontal chromaticity is left as negative through the acceleration). $\gamma_{t r}$ quads were turned on during the transition crossing to maintain longitudinal beam stability. Due to the interference between tune jump quads and $\gamma_{t r}$ quads, six pulses(12 jumps) of tune jump pulses were left off around transition. So only 70 out of 82 horizontal intrinsic resonances were jumped. 
To benefit from the tune jump, the beam particles have to cross the resonance line during the jump. For the given beam parameters (tune jump amplitude, chromaticity, beam momentum spread), about $76 \%$ of the beam will benefit from the tune jumps above $G \gamma=19$. For tune jumps below $G \gamma=12$, more than $76 \%$ of the beam will benefit. Moreover, the benefit portion of beam around transition is smaller, due to the larger momentum spread. Leaving horizontal tune jump quads off around transition has limited disadvantage.

One can not change chromaticity fast enough (in a few ms) to make the tune jump always in the slightly beneficial percentage. With the same sign of chromaticity, it is always true that more particles will benefit from the tune jump in one case (such as $\mathrm{K}-\nu_{x}$ ), but less from the other one (such as $\mathrm{M}+\nu_{x}$ ). With negative chromaticity, one can see that more particles are benefiting from the tune jumps for $K-\nu_{x}$ resonances than the ones for $M+\nu_{x}$. However, since the resonance width is dominated by the spread in $G \gamma$ (Fig. 2), benefit of fast chromaticity change is too small to be considered.

The jump timing determination requires accurate determination of beam energy as a function of ramp time. The beam energy information on the energy ramp comes from measuring the AGS main magnetic field and measuring the beam momentum offset using the radial average from the beam position measuring system. As a cross check, the second set of beam energy information is derived from beam frequency and beam path length. The jump timing is then derived from the beam energy and horizontal tune as function of ramp time.

From these sketch plots, one can estimate how accurate the jump quad timing has to be for the given momentum spread and ramp rate. Although the tune jump duration is $100 \mu \mathrm{s}$, some portion of the beam can still benefit from the tune jump with an offset of jump timing larger than $100 \mu \mathrm{s}$. For FWHM width of 0.04 (typical value after $G \gamma=19$ ) and the ramp rate of $0.117 / \mathrm{ms}$, the FWHM time width of the beam is $0.04 /(0.117 / \mathrm{ms})=0.341 \mathrm{~ms}$. Assuming Gaussian longitudinal distribution, the $\sigma$ of such a distribution should be $145 \mu \mathrm{s}$. For the case below transition, where FWHM of $\mathrm{dp} / \mathrm{p}$ is less than 0.04 , the $\sigma$ should be even smaller. Moreover, the horizontal intrinsic resonances can be treated as isolated resonances due to their spacing and weak strength. The final polarization is a product of Froissart-Stora formula for each resonance crossing. Assuming a Gaussian distribution in the longitudinal dimension, the polarization as a function of overall jump timing shift should be close to a Gaussian distribution. The $\sigma$ of the Gaussian distribution should be around $145 \mu \mathrm{s}$. If there are errors in the individual jump quad timing, the distribution $\sigma$ will be larger.

Final polarization at AGS flattop was actually measured as a function of overall jump timing for $2 \times 10^{11}$ bunch intensity. The measured timing scan curve is shown in Fig. 3 and it clearly demonstrates the benefit on polarization from the tune jump. When the tune jump timing is off by more than $500 \mu \mathrm{s}$ or no particle benefits from the tune jump, and the polarization level is at $63 \%$. When the tune jump timing is at its ideal value, polarization is about $73 \%$. A Gaussian fit shows $\sigma=214 \mu \mathrm{s}$, 


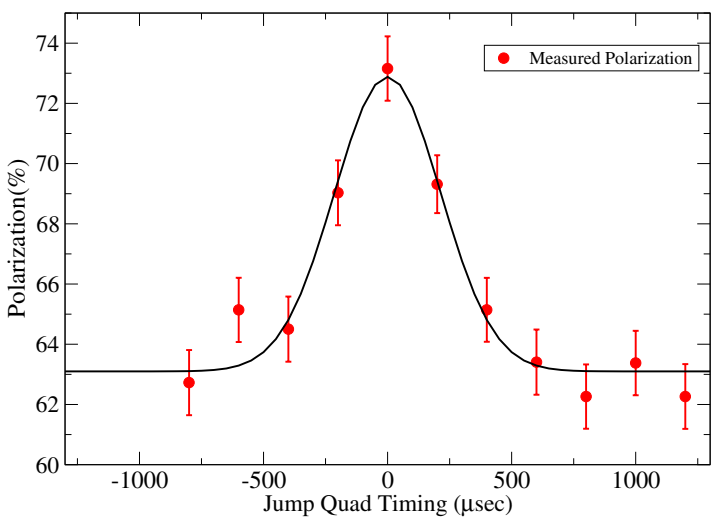

Fig. 3. The measured polarization at AGS extraction as a function of overall jump quad timing. Error bars are statistical errors only. The solid line is a fit of Gaussian distribution with $\sigma=214 \mu s$.

which is slightly larger than the estimation based on the known beam parameters. This implies that there are some tune jump timing errors. Further improvement on tune jump timing is possible.

\section{Conclusions}

Vertical intrinsic resonances and imperfection resonances have been avoided by introducing two partial snakes in the AGS. But the partial snake magnets also tilt the stable spin direction away from the vertical and consequently excite depolarizing resonances associated with horizontal tune, so-called horizontal intrinsic resonances. They are generally weak but could cause accumulated polarization loss if left uncorrected. A modest horizontal tune jump system has been used to overcome these weak but numerous resonances while maintaining the transverse emittances. A relative gain of $15 \%$ polarization has been achieved with the tune jump system. The keys for the system are the accurate control of the jump quad timing and the elimination of any emittance growth. This scheme paves the way to use partial snakes to preserve polarization in the medium energy synchrotrons without the concern of losing polarization due to horizontal intrinsic resonances.

\section{References}

1. Ya. S. Derbenev and A. M. Kondratenko, Part. Accel. 8, 115 (1978).

2. A.D. Krisch, et al., Phys. Rev. Lett. 63, 1137(1989).

3. T. Roser, in Proc. of the 8th International Symposium on High-Energy Spin Physics, Minneapolis, 1988, AIP Conf. Proc. No 187 (AIP, New York,1989), p.1442.

4. H. Huang, et al., Phys. Rev. Lett. 73, 2982 (1994).

5. H. Huang, et al., Phys. Rev. Lett. 99, 154801 (2007).

6. F. Lin, et al., Phys. Rev. ST AB 10, 044001 (2007).

7. H. Huang, et al., Proc. of IPAC14, p.2112. 